\title{
ON A THEORY OF OBJECTS BASED ON A SINGLE AXIOM SCHEME
}

\author{
KATUZI ONO
}

\section{Introduction}

There are some fundamental mathematical theories, such as the Fraenkel set-theory ${ }^{11}$ and the Bernays-Gödel set-theory ${ }^{2}$, in which, I believe, all the actually important formal theories of mathematics can be embedded. Formal theories come into existence by being shown their consistency. As far as this is admitted, not all the axioms of set theory are necessary for a fundamental mathematical theory. The fundierung axiom is proved consistent by $v$. Neumann ${ }^{3)}$, the axiom of extensionality is proved consistent by Gandy ${ }^{4}$, and even the axiom of choice is proved consistent by Gödel ${ }^{5}$. Although it is not evident that a set-theory does not cease from being a fundamental theory of mathematics after abandoning these axioms all at once, the theory must be enough for being a fundamental theory of mathematics without some of them.

The object theory I am going to introduce here is intended to be a fundamental theory of such kind. The system has only one primitive notion " $\in$ " (the membership-relation) and assumes only one axiom scheme.

Let us now explain the practical meaning of the only axiom scheme of my system. To begin with, let us consider the axiom of replacement of the Fraenkel set-theory. The axiom asserts for any set $h$ and for any unique mapping $\Delta(x \Delta y$ is unique regarding it as a mapping $y$ to $x$ ) existence of a set $k$ formed by all the $\Delta$-images (images by $\Delta$ ) of members of $h$. Starting from this assertion, we strengthen it in the following two steps. Namely, in the first step, we replace "members of $h$ " by "sub-sets of members of $h$ ". In the second step, we assume existence of a set $p$ generated recursively by the

Received September 17, 1964.

1) Fraenkel [1], [2].

2) Bernays [1], Gödel [1], See also v. Neumann [1].

3) v. Neumann [2].

1) Gandy [1].

5) Gödel [1]. 
mapping $h$ to $k$ starting from any given set $m$.

Thus the only àssumption of my new system turns out to be the following:

(A) For any $m$ and for any unique mapping $\Delta$, there is $p$ such that, for any $h$ in $p$, there is $k$ in $p$ formed by all the $\Delta$-images of sub-objects (sub-sets) of members of $h$.

Inclusion and equality can be defined as usual by extension as follows :

$$
\begin{aligned}
& x \subseteq y{ }^{6}(s)(s \in x \rightarrow s \in y), \\
& x=y ₹(s)(s \in x \equiv s \in y) .
\end{aligned}
$$

Any mapping $x \oplus y(y$ to $x$ ) is expressed by a relation between $x$ and $y$, i.e. a sentence on two free variables $x$ and $y$. The notion of sentences can be defined meta-theoretically as usual. The condition for that a relation $\emptyset$ denotes a unique mapping can be now stated in our system. However, I prefer a more agreeable way to state the assumption (A) by making use of the comprehension operator $\{\Gamma)^{\tau}$, which operates on any binary relation $\Gamma$ and gives a new binary relation defined by

$$
x\{\Gamma) y ₹(s)(s \in x \equiv s \Gamma y) .
$$

Namely, $x\{\Gamma) y$ denotes that $x$ is a set formed by all the objects $s$ bearing the relation $s \Gamma y$ to $y$. By virtue of the comprehension operator, descriptions can be simplified. Equality = can be expressed as $\{\in$ ). Moreover, any relation of the form $\{\Gamma)$ is proved to be unique ${ }^{8)}$, so

$$
(m)(\exists p)\left(m \in p \wedge(h)_{p}(\exists k)_{p} k\{\langle\Gamma) \subseteq \in) h\right)^{q)}
$$

is a special case of (A). It is enough for my system to assume only this special case of $(A)^{101}$.

6) We use the notation $\mathfrak{A} \vDash \mathfrak{B}$ to denote that $\mathfrak{P}$ is defined by $\mathfrak{B}$, i.e. that $\mathfrak{A}$ stands for $\mathfrak{B}$, usually a longer expression than $\mathfrak{X}$.

7) The symbol " $\{\cdot$ )" was introduced in Ono [3], and the name "comprehension operator" was introduced in Ono [4].

8) Any relation of the form $\{\Gamma)$ is unique in the sense $(x)(y)(z)(x\{\Gamma) z \wedge y\{\Gamma) z \rightarrow x=y)$, which is provable without any assumption.

9) Any formula of the forms $(h)_{p} \mathfrak{Y}(h),(\exists k)_{p} \mathfrak{F}(k)$ stands for $(h)(h \in p \rightarrow \mathscr{Q}(h)),(\exists k)(k \in p$ $\wedge \mathfrak{F}(k))$, respectively. The relation product is defined by $x(\Gamma \Delta) y=(\exists z) x \Gamma z \Delta y$. As the product is associative, parentheses denoting the order of combination are usually omitted.

10) It does not seem provable that every unique relation $\Delta$ is expressible in the form $\{\Gamma$ ). But, without any assumption, it is provable that every unique relation expressible in the form $=\Delta$ can be expressed in the form $\{\Gamma$ ). See Ono [4]. 
Every proposition of the form $\left(A^{\prime}\right)$ is provable in the Fraenkel set-theory. As $\left(A^{\prime}\right)$ is the sole assumption of my system, it is evidently consistent relative to the Fraenkel set-theory. In my system, none of the sum-set axiom, the aussonderung axiom, the fundierung axiom, the extensionality axiom, and the choice axiom seems to be provable. However, all the axioms of the Fraenkel set-theory except the choice axiom are provable in a certain domain, the domain of sets, which is definable in my formal system. Accordingly, one may also follow the Gödel theory ${ }^{11}$ to prove consistency of the choice axiom with respect to my system with suitable modification, but I do not carry it out in this work. Here I explain only how the notion of sets can be defined in my formal system and how the axioms of the Fraenkel set-theory other than the choice axiom are provable in the domain of sets.

I have tried already a few approaches of the same kind. In my system $\mathrm{OF}$, the satellite relation " $\sigma$ " must be defined before stating its axiom scheme. The axiom scheme of $\mathbf{O F}$ is really simple if the notion of satellite relation is assumed to be known, but the notion seems too complicated ${ }^{12}$. My new system has really the same range as the system OF. My apology for introducing this new system is in the matter of style. I believe that $\left(A^{\prime}\right)$ is a form more agreeable than the axiom scheme of OF.

It was not necessary to change very much the embedding process of the Fraenkel set-theory without the choice axiom in of for embedding the same set-theory in my new system. In this work, I will rather give an informal exposition of my system and explain the leading idea of constructing a settheory in it. Detailed formal description will be given elsewhere.

In (1), I will give a precise exposition of the system. Membership " $\in$ "

11) Gödel [1].

12) My former three works Ono [1], [2], and [4] are on the same line. In $\mathrm{OZ}$ (the system introduced in [2]) and in OF (the system introduced in [4]), I used the satellite relation $\sigma$. In both systems, I have defined the satellite relation $\sigma$ after defining "inclusion $\subseteq$ " and "unit-object relation $\nu$ " ( $x \nu y$ denotes that $x$ is a unit object (unit set) of $y$ ) so that $\sigma$ is the minimal transitive relation which is larger than $\in, \subseteq$, and $\nu$. (Any relation $\Gamma$ is called larger than another relation $\Delta$ (or $\Delta$ implies $\Gamma$ ) if and only if $(x)(y)(x \Delta y \rightarrow x \Gamma y$ ) holds.) As it was convenient to define "inclusion" and "unit-object relation" somewhat differently in these two systems, so the notion of satellite of OF turned out to be more complicated than that of $\mathbf{O Z}$.

Just as this new system, each of my two former systems $O Z$ and $O F$ is based on a single axiom scheme. Quine's system is also of the same kind. See Quine [1], [2]. 
is the only primitive notion of the system. Capital Latin letters are employed as object variables. 'Capital Greek letters are employed as meta-theoretical symbols for binary relations. The axiom scheme of the system can be introduced by using these outfits only.

In (2), some fundamental properties are written down for reference' sake. Some of them are definitions in any systems of the same outfits and the others are theorems or meta-theorems provable without any assumption.

In (3), some general properties of my new system are proved. In (4), the descendent relation " $\delta$ " is defined and several properties of the relation are proved.

In (5) and in (6), the notions of normality ${ }^{13)}$ and regularity are introduced, and some theorems concerning each of these notions are described, respectively.

In (7), the domain of sets is defined as the domain of normal and regular objects, and all the axioms of the Fraenkel set-theory except the choice axiom are shown provable in the domain of sets. To make descriptions simpler, set variables denoted by lower case Latin letters are introduced.

\section{(1) The system}

$(1.1 \mathrm{~S})^{14}$ Membership " $\in$ " is the only primitive notion of the system. Variables for objects in general are denoted by capital Latin letters.

$(1.2 \mathrm{R})^{15)}$ Together with the usual logical symbols $\wedge, \vee, \rightarrow, \rightarrow, \equiv,()$, ( $\exists$ ), quantifiers of specified ranges having the forms $(X)_{M} \mathfrak{U}(X)$ and $(\exists X)_{M} \mathfrak{A}(M)$ are also employed ${ }^{16 !}$.

The notion of open sentences can be introduced as usual. Open sentences on two variables $X$ and $Y$ are denoted in the form $X I Y Y$. Capital Greek letters are for binary relations as meta-logical variables. The variables $X$ and $Y$ need not really occur in the open sentence $X I Y$. Moreover, some variables other than $X$ and $Y$ may occur in $X \Gamma Y$ as parameters. In fact, the variable $X$ and $Y$ of $X I Y$ are given just to call for notice that the relation $X \Gamma Y$ is a relation between $X$ and $Y$ defined by a suitable open sentence.

13) Normality was called total normality in Ono [4].

14) Numbers of the form $(\boldsymbol{m}, \boldsymbol{n})$ are for theorems and meta-theorems. Numbers of the form $(m, n S)$ are for illustrations just stated.

15) Numbers of the form $(m, n R)$ are for remarks.

16) See Foot-note 9). 
Notations for relation products and comprehension operators are also employed ${ }^{17}$.

$(1.3 \mathrm{D})^{18)}$ Inclusion $\subseteq$ is defined as usual by

$$
X \subseteq Y ₹(S)(S \in X \rightarrow S \in Y) \text {. }
$$

Any object $X$ satisfying $X \subseteq Y$ is called a sub-object of $Y$.

$$
(1.4 \mathrm{~A})^{19)} \quad(M)(\exists P)\left(M \in P \wedge(H)_{P}(\exists K)_{P} K\{\{\Gamma) \subseteq \in) H\right)
$$

is the only axiom scheme of the system, where $P$ and $K$ do not occur in $I^{, 201}$.

\section{(2) Some fundamental notions and some fundamental properties provable without any assumption}

\section{In this paragraph, $(1.4 \mathrm{~A})$ is not assumed.}

The followings are fundamental notions and fundamental properties of any system having the notion $\in$. Most of them are already described in my former work $^{21)}$. I write down them here, mostly for reference' sake.

(2.1) $(X) \mathfrak{A}(X) \rightarrow(X)_{M} \mathfrak{\mathscr { A }}(X)$ and $(\exists X)_{M} \mathfrak{A}(X) \rightarrow(\exists X) \mathfrak{A}(X)$.

(2.2 D) $X=Y ₹ X\{\in) Y$.

(2.3) Equality $=$ is reflexive, symmetric, and transitive.

(2.4 D) Any relation $\Gamma$ is called left (or right) invariant if and only if it is equivalent ${ }^{23)}$ to $=\Gamma$ (or $\Gamma=$ ). $\quad \Gamma$ is called invariant if and only if it is right invariant as well as left invariant.

(2.5) $\Gamma$ is left (or right) invariant if $=\Gamma$ (or $\Gamma=$ ) implies $^{231} l$ :

(2.6) $\Gamma \Delta$ is left (or right) invariant if $I$ (or $\Delta$ ) is so. It is invariant if $\Gamma$ is left invariant and $\Delta$ is right invariant.

(2.7) If $\Gamma$ and $\Delta$ are both left (or right) invariant, then $\Gamma \wedge \Delta$ as well as $\Gamma \vee \Delta^{21)}$ is also left (or right) invariant.

17) See Foot-notes 9) and 7 ).

18) Numbers of the form $(m, n \mathrm{D})$ are for definitions.

19) Numbers of the form $(m, n A)$ are for axioms and axiom schemes.

20) We say that $S$ does not occur in $\Gamma$ if $S$ does not occur in some open sentence of the form $X \Gamma Y$ (i.e. in its defining open sentence).

21) Ono [4].

22) $\Gamma$ is said to be equivalent to $\Delta$ if and only if $(X)(Y)(X \Gamma Y \equiv \mathrm{X} \Delta Y)$.

23) $T$ is said to imply $\Delta$ if and only if $(X)(Y)(X \Gamma Y \rightarrow X \Delta Y)$.

24) $X(\Gamma \wedge \Delta) Y \mp \cdot X \Gamma Y \wedge X \Delta Y$ and $X(\Gamma \vee \Delta) Y \bar{₹} X \Gamma Y \vee X \Delta Y$. 
(2.8) Any binary relation of the form $\langle\Gamma\rangle$ is left invariant.

(2.9) For any right invariant $\Gamma,\{\Gamma)$ is invariant.

(2.10) $\{\Gamma)$ is unique ${ }^{25)}$.

(2.11) $\in$ is right invariant.

$(2.12) \subseteq$ is reflexive and transitive.

(2.13) $=$ is equivalent to $\subseteq \wedge \supseteq{ }^{26)}$.

(2.14) $\subseteq$ is invariant.

(2.15) $X\{=) Y$ implies $Y \in X$.

(2.16) $X\{\Gamma) Y \rightarrow X\{=\in) X$ holds for any left invariant $I$.

(2.17) $Y\{=\in) X$ implies $Y\{=\in) Y$.

(2.18) Any unique and left-invariant relation $\Delta$ can be expressed in the form $\{\Gamma)$.

This meta-theorem is not trivially provable. A formal proof of the theorem is given in Ono [4]-(2.31). Although I did not expressly remarked there that the proof is valid for any system having the $\in$ notion, one can check it without difficulty.

(2.19 D) $\phi((X) ₹(S) S \notin X$.

Any object $X$ satisfying $\phi(X)$ is called a null-object.

(2.20) $\quad(X)(Y)(\phi(X) \rightarrow X \subseteq Y)$.

Hereafter, throughout this work, I assume $(1.4 \mathrm{~A})$.

(3) Object theory

In this chapter, some remarkable aspects of the theory of objects in the system are described.

The following (3.1) and (3.2) follow immediately from (1.4 A) and (2.1).

(3.1) $(M)(\exists P) M \in P$.

(For any object $M$, there is an object containing $M$ ).

25) See Foot-note 8 ).

26) $\supseteq$ denotes the inverse relation of $\subseteq$ as usual, 
(3.2) $(H)(\exists K) K\{\langle\Gamma) \subseteq €) H$.

(For any object $H$ and for any relation $\Gamma$, there is an object $K$ formed by all the $\{\Gamma)$-images, naturally $\{\Gamma)$ being unique by $(2.10)$, of sub-objects of members of $H$ ).

Theorems (3.3)-(3.6) are not trivially provable, but their proofs can be carried out quite in parallel with proofs of these theorems in OF. I will sketch the proofs very shortly.

(3.3) $(M)(\rightarrow(T) T \in M)$.

(No object contains all the objects.)

Proof. If there were an object $M$ which contains all the objects, then, by virtue of (3.2), there would be another object $P$ satisfying $P\{(\Gamma) \subseteq \in) M$, where $\Gamma$ is a relation defined by $X \Gamma Y ₹ X \in Y \notin Y$. For this $P$, two contradictory propositions $P \in P$ and $P \notin P$ are provable by a pretty long deduction employing (2.3), (2.8), and (2.16).

\section{(3.4) $(\exists X) \phi(X)$.}

( There is a null object.)

Proof. For any object $M$, there is an object $X$ such that $X\{\{\Gamma) \subseteq \in) M$ by (1.4 A), where $\Gamma$ is a relation defined by $S \Gamma T ₹(S \in T \rightarrow S \in T)$. According to (3.3), $X$ is a null object.

(3.5) $(H)(\exists K) K\{\{\Gamma) \in) H . \quad(H$ should not occur in $\Gamma$.)

(For any object $H$ and for any relation $\Gamma$, there is an object $K$ formed by all the $\{\Gamma)$-images, naturally $\{\Gamma)$ being unique by $(2.10)$, of members of $H$. This is a special case of the axiom of replacement.)

Proof. For any object $H$ and for any relation $\Gamma$, there is an object $K$ satisfying $K\{(\Delta) \subseteq \in) H$ by $(3.2)$, where $\Delta$ is a relation defined by $X \Delta Y ₹(Y \in H \rightarrow X \Gamma Y)$. For this $K, K\left\{\left\{I^{\prime}\right) \in\right) H$ is provable by making use of (3.3) and (2.12).

(3.6) $(M)(\exists P) P\{=(\Gamma \wedge \in)) M . \quad(P$ should not occur in $\Gamma$.

(For any object $M$ and for any relation $\Gamma$, there is an object $P$ formed by all the objects each being equal to some member $X$ of $M$ satisfying $X \Gamma M$. This is the aussonderung axiom if the extensionality axiom is assumed.) 
Proof. For any object $M$ and for any relation $\Gamma$, there is an object $P$ satisfying $P\{\{\Delta\} \in) M$ by $(3.5)$, where $\Delta$ is a relation defined by $X \Delta Y$ ₹ $(Y \Gamma M$ $\rightarrow X \in Y)$. For this $P, P\{=(\Gamma \wedge \in)) M$ is provable by making use of (3.3).

I will prove Theorems (3.7), (3.9)-(3.11) something more in details, as their proofs in this system show somewhat different features from their proofs in $\mathbf{O F}$.

(3.7) $(M)(\exists P) P\{\subseteq) M$.

(For any object $M$, there is an object formed by all the sub-objects of $M$. Any object $X$ satisfying $X\{\subseteq) Y$ is called a power-object of $Y$. The power-set axiom.)

Proof. For any object $M$, there is an object $Z$ containing $M$ by (3.1). By (3.2), there is an object $P$ satisfying $P\{\{\Gamma) \subseteq \in) Z$, where $\Gamma$ is a relation defined by $S \Gamma T ₹ S \in T \in M$. This object $P$ satisfies $P(\subseteq) M$. To show this, one can prove easily $(U)(U \in P \rightarrow U \subseteq M)$. On the other hand, any sub-object $U$ of $M$ satisfies $U\{\Gamma) U$, so $U\{\Gamma) U \subseteq M \in Z$ holds. Hence, $U$ is a member of $P$ by definition.

(3.8 D) $Z\{X, Y\} ₹(S)(S \in Z \equiv \cdot S=X \vee S=Y)$.

Any object $Z$ satisfying $Z\{X, Y\}$ is called a pair-object of $X$ and $Y$.

(3.9) $(X)(Y)(\exists Z) Z\{X, Y\}$.

(For any two objects, there is a pair-object of them.)

Proof. To prove the theorem, I will prove existence of an object $W$ which contains at least two objects $U$ and $V$. This can be done by taking a null object $U$ by (3.4), its power-object $V$, and a power-object $W$ of $V$ by (3.7), $W$ contains surely $U$ and $V$, and $V$ is not a null-object because it contains the null object $U$ according to (2.20).

Now, for any $X$ and $Y$, one can define a relation $\Delta$ such that the relation (d) maps every null object to $X$ and every object other than null objects to $Y$. Namely, $\Delta$ is a relation defined by $S \Delta T ₹ \cdot(T=U \wedge S \in X) \vee(T \neq U \wedge S \in Y)$. Hence, if one take an object $Z$ satisfying $Z\{\{\Delta) \in) W$ by (3.5), $Z$ satisfies $Z\{X, Y\}$.

$(3.10)(M)(\exists P) P\{=\in \in) M$. 
(For any object $M$, there is an object $P$ formed by all the objects, each being equal to a member of a member of $M$. This is the sum-set axiom, if the extensionality axiom is assumed.)

Proof. For any $M$, one can take such an object $N$ that $P\{=\in \in) N$ is equivalent to $P\{=\in \in$ ) $M$ for every $P$ (in other words $(S)(S=\in \in N \equiv S=\in \in M$ ) holds) and that every member $T$ of $N$ satisfies $T\{=\in) T$. Any object satisfy. ing $N\{\{=\in) \in) M$ taken by (3.5) is proved to be an object of this kind by making use of (2.3), (2.6), (2.8), and (2.16).

Now, there is an object $P$ satisfying $P\{\{\Gamma) \subseteq \in) N$ by $(3.2)$, where $\Gamma$ is a relation defined by $S \Gamma T \geqslant((\exists Z) T\{=) Z \rightarrow S \in \in T)$. I will show that this $P$ satisfies $P\{=\in \in) N$. At first, I will show that any member $R$ of $P$ satisfies $R=\in \in N$. Namely, for any member $R$ of $P$, there are two objects $U$ and $V$ satisfying $R\{\Gamma) U \subseteq V \in N$ by definition. By reductio ad absurdum, existence of an object $Z$ satisfying $U\{=) Z$ can be proved taking (3.3) into account. This $Z$ can be proved to be equal to $R$. Because $Z$ is a member of $U$ by (2.15), $R=Z \in V \in N$ holds. Conversely, any object $R$ which is equal to a member $Z$ of a member $V$ of $N$ is a member of $P$. Because the member $V$ of $N$ satisfies $V\{=\in) V, R$ is a member of $V$. Now, take an object $U$ satisfying $U\{=) R$ by (3.9), then $(S)(S \Gamma U \equiv S \in \in U)$ holds by definition of $\Gamma$. By making use of (2.15), one can prove $R\{\Gamma) U$ without difficulty. Moreover, $U$ is a sub-object of $V$ because $U\{=) R$ and $V\{=\in) V$ hold. Hence, $R\{\Gamma) U \subseteq V \in N$.

(3.11) $(M)(\exists P)\left(M \in P \wedge(H)_{P}(\exists K)_{P} K\{=) H\right)$.

(For any object $M$, there is an object $P$ which contains $M$ and also contains every unit-object ${ }^{2 \pi}$ ) of any member of itself.)

Proof. For any $M$, one can take a unit object $N$ of $M$ and a unit object $L$ of $N$ by (3.9). By technical reason, I will prove existence of an object $Q$ containing $L$ and satisfying $(H)_{Q}(\exists K)_{Q} K\{=) H$. If existence of such $Q$ is shown, then the theorem can be proved as follows: One can take an object $P$ satisfying $(H)(H \in P \equiv \cdot H=M \vee H=N \vee H=\in Q)$ by employing (2.3), (3.9), and (3.10). This $P$ surely contains $M$ by (2.3), and it can be proved to satisfy $(H)_{P}(\exists K)_{P} K\{=) H$ by making use of (2.3) and (2.9).

Existence of such $Q$ is proved as follows: By (1.4 A), there is an object

27) Any object $X$ satisfying $X\{=) Y$ is çalled a unit object of $Y$, 
$R$ satisfying $L \in R$ and $(H)_{R}(\exists K)_{R} K\{\{\Gamma) \subseteq \in) H$, where $\Gamma$ is a relation defined by $S \Gamma Y ₹((\exists W) Y\{\stackrel{\prime}{=}) W \rightarrow S=Y)$. For this $\Gamma$, one can prove that $K\{\{\Gamma) \subseteq \in) H$ implies $K\{=) H$ as far as $H$ is a unit object of a unit object of some object, by making use of $(2.3),(2.8),(2.9),(2.10),(2.12),(2.15)$, and especially (3.3). Now, according to (3.6), there is an object $Q$ satisfying $Q\{=(\Delta \wedge \in)) R$, where $\Delta$ is a relation defined by $X \Delta Y ₹(\exists J) X\{=)\{=) J$. By (2.3), (2.8), and (2.9), $Q$ is proved to be an object formed by all the members of $R$, each being a unit-object of a unit-object. Hence, $(H)_{Q}(\exists K)_{Q} K\{=) H$ holds.

\section{(4) Descendent relation}

The notion of the descendent relation $\delta$ in this system is the same as that of the descendent relation in OF. Most remarkable difference between my new system and the system OF may be that existence of an object formed by all the descendents (see $(4.1 \mathrm{D})$ ) of any object is easily provable in $\mathrm{OF}$, whereas it does not seem provable in the new system. By this circumstance, I had to prove Theorem (4.6) in a complicated manner by making use of Lemma $(4.5 \mathrm{~L})^{36)}$ compared with the manner of the proof for the corresponding theorem in $\mathbf{O F}^{29)}$.

(4.1 D) Just as in OF, the descendent relation $\delta$ is defined by

$$
X \delta Y ₹(P)((S)(S \in \in P \rightarrow S \in P) \wedge Y=\in P \cdot \rightarrow X=\in P) .
$$

Any object $X$ satisfying $X \delta Y$ is called a descendent of $Y$.

By making use of (2.3), Theorems (4.2)-(4.4) are easily provable.

(4.2) The descendent relation $\delta$ is reflexive and transitive.

(4.3) Equality $=$ implies the descendent relation $\delta$.

(4.4) The membership relation $\in$ implies the descendent relation $\delta$.

For any object $Y$ in general, I can prove $(\exists Z) Z\{\delta) Y$ only under a certain condition (See $(4.5 \mathrm{~L})$.), but the condition does not cause any essential difficulty for proving the fundamental property (4.6) of the descendent relation.

$(4.5 \mathrm{~L}) \quad(\exists P)((S)(S \in \in P \rightarrow S \in P) \wedge Y=\in P) \rightarrow(\exists Z) Z\{\delta) Y$.

(For any $Y$, there is an object formed by all the descendents of $Y$, as far

28) Numbers of the form $(m, n L)$ are for lemmas.

29) Ono [4], Theorem (7.7), 
as there is an object $P$ containing all the members of its members and an object equal to $Y$.)

Proof. Assume that one can take $P$ such that $(S)(S \in \in P \rightarrow S \in P)$ and $Y=\in P$ hold. For this $P$, take an object $Z$ by (3.6) satisfying $Z\{=(\Gamma \wedge \in)) P$, where $\Gamma$ is a relation defined by $S \Gamma T \geqslant S \delta Y . \quad Z\{\delta) Y$ can be proved without difficulty by making use of (2.3), (4.1 D), (4.2), and (4.3).

(4.6) $(X)(Y)(X \delta Y \equiv \cdot X \delta \in Y \vee X=Y)$.

(Any object $X$ is a descendent of another object $Y$ if and only if $X$ is a descendent of some member of $Y$ or $X$ is equal to $Y$.)

Proof. For any $X$ and $Y, X \delta \in Y$ as well as $X=Y$ implies $X \delta Y$ according to $(4.2),(4.3)$, and (4.4).

Now, I prove conversely that $X \delta Y$ implies $X \delta \in Y \vee X=Y$. If $Y$ contains a member $U$ for which no object $P$ satisfies $(S)(S \in \in P \rightarrow S \in P) \wedge U=\in P$, then any object, especially $X$, is evidently a descendent of $U$, so $X$ satisfies $X \delta U \in Y$. Accordingly, without loss of generality, I can assume for every member $U$ of $Y$ existence of an object $P$ satisfying $(S)(S \in \in P \rightarrow S \in P) \wedge$ $U=\in P$. By virtue of $(4.5 \mathrm{~L})$, there is an object $Z$ satisfying $Z\{\delta) U$ for every member $U$ of $Y$.

By (3.5), take an object $Q$ such that $Q\{\{\delta) \in) Y$, and thereafter take an object $R$ satisfying $R\{=\in \in) Q$ by (3.10). This $R$ can be proved to satisfy $R\{\delta \in) Y$ by (2.3), (4.2), and (4.3). By virtue of (3.9) and (3.10), there is an object $P$ consisting of all the objects equal to $Y$ in addition to all the members of $R$. In fact, one has only to take the objects $Z, B$, and $P$ in succession such that $Z\{=) Y, B\{R, Z\}$, and $P\{=\in \in) B$. For this $P,(S)(S \in P$ $\equiv \cdot S \delta \in Y \vee S=Y$ ) can be proved by taking (2.3), (4.2), and (4.3) into consideration. Moreover, $(S)(S \in \in P \rightarrow S \in P)$ is provable by (2.3) and (4.2), and $Y=\in P$ is provable by (2.3). Hence, the descendent $X$ of $Y$ must be equal to some member $W$ of $P$.

Because the member $W$ of $P$ satisfies $W \delta \in Y \vee W=Y$, so $X \delta \in Y \vee X=Y$ holds too according to (2.3), (4.2), and (4.3).

\section{(5) Normality}

Normality is defined just as in OF. Theorems (5.2)-(5.4) can be proved very easily. 
$(5.1 \mathrm{D}) \quad \nu(X) ₹(S)(S \delta X \rightarrow S\{=\epsilon) S)$.

(Any object $X$ sátisfying $\nu(X)$ is called normal ${ }^{30)}$.)

(5.2) $(X \mid \phi(X) \rightarrow \nu(X))$.

(Any null object is normal.)

Proof. By (4.6).

(5.3) $(X)(Y)(X \delta Y \wedge \nu(Y) \cdot \rightarrow \nu(X))$.

(Any descendent of a normal object is also normal.)

Proof. By (4.2).

(5.4) $(X)\left((S)_{X} \nu(S) \wedge X\{=\in) X \cdot \rightarrow \nu(X)\right)$.

(Any object $X$ consisting of normal objects and satisfying $X\{=\in$ ) $X$ is also normal.)

Proof. By (2.3), (2.11), (2.16), and (4.6).

\section{(6) Regularity}

Regularity is also defined just as in OF. Theorems (6.2)-(6.4) can be proved quite similarly as their corresponding theorems in OF.

(6.1 D) The regularity relation $\rho$ is defined at first, and regularity is defined thereafter by making use of the regularity relation.

$$
\begin{aligned}
& M \rho N ₹(X)(X \delta M \wedge X \in N \cdot \rightarrow X \ni \in N)^{31)}, \\
& \rho(M) ₹-M \rho \ni \delta M .
\end{aligned}
$$

Any object $M$ satisfying $\rho(M)$ is called regular.

(6.2) $(X)(\phi(X) \rightarrow \rho(X))$.

(Any null object is regular.)

Proof. By (4.6).

(6.3) $(X)(Y)(X \delta Y \wedge \rho(Y) \cdot \rightarrow \rho(X))$.

(Any descendent of a regular object is also regular.)

Proof. By (2.3), (3.6), (4.2), and (4.4).

$(6.4) \quad(M)\left((X)_{u} \rho(X) \rightarrow \rho(M)\right)$.

30) See Foot-note 13).

31) $\ni$ is naturally the inverse relation of $€$. 
(Any object consisting of regular objects is also regular.)

Proof. By (4.2), (4.4), and (4.6).

\section{(7) Set theory}

The set notion is defined just as in OF. I introduce also set variables. By making use of set variables, I introduce some set-theoretical notions.

$(7.1$ D) $\$(X) ₹ \cdot \nu(X) \wedge \rho(X)$.

Any object $X$ satisfying $\$(X)$ is called a set. Accordingly, any object is a set if and only if it is normal and regular.

(7.2 R) Set variables are used to denote sets. Lower case Latin letters are used for set variables. Namely, for a free set-variable $x, \$(x)$ is tacitly assumed. For a bound set-variable $x$, the following definitions hold.

$$
\begin{aligned}
& (x) \mathfrak{U}(x) ₹(X)(\$(X) \rightarrow \mathfrak{U}(X)), \\
& (\exists x) \mathfrak{U}(x) ₹(\exists X)(\$(X) \wedge \mathfrak{U}(X)) .
\end{aligned}
$$

Set-theoretical inclusion $\subseteq$, set-theoretical equality $\overline{\bar{c}}$, set-theoretical relation product $\Gamma^{*} \Delta$, set-theoretical comprehension-operator $\langle\Gamma)_{0}$, and set-theoretical pairing $Z\{X, Y\}_{\circ}$ are defined as follows:

$$
\begin{aligned}
& X \subseteq Y ₹(s)(s \in X \rightarrow s \in Y), \\
& X \bar{\circ} ₹(s)(s \in X \equiv s \in Y), \\
& X \Gamma^{*} \Delta Y ₹(\exists s) X \Gamma s \Delta Y, \\
& X\{\Gamma)_{\circ} Y ₹(s)(s \in X \equiv s \Gamma Y), \\
& Z\{X, Y\}_{\circ} ₹(s)(s \in Z \equiv \cdot s \overline{\overline{0}} X \vee s \overline{\bar{\sigma}} Y)
\end{aligned}
$$

Following Theorems (7.3)-(7.8) can be proved very easily.

(7.3) $(X) \mathfrak{A}(X) \rightarrow(x) \mathfrak{U}(x)$ and $(\exists x) \mathfrak{U}(x) \rightarrow(\exists X) \mathfrak{A}(X)$.

(7.4) $(X)(\phi(X) \rightarrow \$(X))$ and $(\exists x) \phi(x)$.

(Any null object is a set, and there is a null set ${ }^{32}$.)

Proof. By (3.4), (5.2), and (6.2).

(7.5) $(M)\left((X)_{M} \$(X) \wedge M\{=\in) M \cdot \rightarrow \$(M)\right)$.

(Any object $M$ consisting of exclusively sets and satisfying $M\{=\in$ ) $M$ is

32) I call here a set being a null-object a null-set. 
a set.)

Proof. By (5.4) and (6.4).

(7.6) $(x)(Y)(Y \delta x \rightarrow \$(Y)$.

(Any descendent of a set is also a set.)

Proof. By (5.3) and (6.3).

(7.7) $(x)(Y)(x \subseteq Y \equiv x \subseteq Y)$.

(Any set is set-theoretically included in an object if and only if it included in the object.)

Proof. By (4.4), (7.3), and (7.6).

(7.8) $(x)(y)(x \overline{\bar{\sigma}} y \equiv x=y)$.

(Two sets are set-theoretically equal to each other if and only if they are mutually equal. )

Proof. By (7.7).

Now, I will show in the following that all the axioms of the Fraenkel settheory except the choice axiom are provable in my system with respect to the set-theoretical notions introduced in (7.2 R).

(7.9) As the set-theoretical equality $\overline{\bar{\sigma}}$ is so defind that $(x)(y)(x \overline{\bar{\sigma}} y \equiv$ $(s)(s \in x \equiv s \in y))$ holds, the extensionality axiom can be described as

$$
(x)(y)(z)(x \overline{\bar{\sigma}} y \rightarrow(x \in z \equiv y \in z)) .
$$

This can be expressed by my notation shortly as

$$
(z) z\{\overline{\bar{o}} * \in)_{0} z \text {. }
$$

Proof of the extensionality axiom. By virtue of (2.3) and (7.8), (s) $(s \in z \rightarrow$ $\left.s \overline{0}^{*} \in z\right)$ holds. To show conversely $(s)(s \overline{0} * \in z \rightarrow s \in z)$, take any $s$ satisfying $s z^{*} \in z$. By virtue of (7.3) and (7.8). $s=\in z$ holds. Since the set $z$ is normal by definition, so $s$ must be a member of $z$.

(7.10) The pair-set axiom can be described as

$$
(x)(y)(\exists z) z\{x, y\}_{\circ} \text {. }
$$

Proof of the pair-set axiom. For any $x$ and $y$, there is an object $Z$ satisfying $Z(x, y)$ by (3.9). This $Z$ is a set according to $(7.5)$, because $(T)_{Z} \$(T)$ is 
provable by making use of (4.3) and (7.6) and $Z\{=\epsilon) Z$ is provable by making use of (2.3) and (7.5). Moreover, $Z\{x, y\}$ implies $Z(x, y\}$ 。 by (7.3) and (7.8).

(7.11) The power-set axiom can be described as

$$
(x)\left(\exists y \mid y\{\subseteq)_{0} x\right.
$$

Proof of the power-set axiom. For any $x$, take an object $U$ such that $U\{\subseteq) x$ by (3.7), and next take another object $Y$ such that $Y\{=(\Gamma \wedge \in)) U$ by (3.6), where $\Gamma$ is a relation defined by $S T T ₹ \$(S)$. For this $Y,(Z)_{Y} \$(Z)$ is provable by making use of (4.3) and (7.6), and $Y\{=\in) Y$ is provable by (2.3). Hence, $Y$ is a set by (7.5). By taking (2.3), (7.3), and (7.7) into consideration, one can prove $Y(\subseteq)$ ) $x$.

(7.12) The sum-set axiom is described as

$$
(x)(\exists y) y\left\{\in^{*} \in\right)_{0} x .
$$

Proof of the sum-set axiom. For any $x$, take an object $Y$ such that $Y\{=\epsilon \in) x$ by (3.10). For this $Y,(Z)_{Y} \$(Z)$ is easily provable by making use of (4.2), (4.3), (4.4), and (7.6), and $Y\{=\equiv) Y$ is provable by (2.3). Hence, by (7.5), $Y$ is a set. $Y\left\{\in^{*} \in\right)_{\diamond} x$ is provable without difficulty by taking (2.3), (4.2), (4.4), (7.3), and (7.6) into consideration.

(7.13) The aussonderung axiom can be described as

$$
(x)(\exists y) y\{\Gamma \wedge \in)_{0} x .
$$

Here, the relation $S \Gamma T$ could be assumed expressible in terms of set variables only except for the free variables $S$ and $T$. This assumption is really necessary for proving the theorem.

Proof of the aussonderung axiom. For any $x$, take an object $Y$ such that $Y\{=(\Gamma \wedge \in)) x$ by (3.6). For this $Y,(Z)_{Y} \$(Z)$ is provable by making use of (4.2), (4.3), (4.4), and (7.6), and $Y\{=\in) Y$ is provable by making use of (2.3). Hence, $Y$ is a set by (7.5). $Y\{\Gamma \wedge \in)_{0} x$ is provable by taking into account Theorems (2.3), (4.3), and (7.6) together with assumption for $\Gamma$ that $S T T$ can be expressed in terms of set variables only except for $S$ and $T$.

(7.14) The axiom of replacement can be described as 


$$
(x)(\exists y) y\{\Theta * \in))_{0} x
$$

where $\Theta$ is a set-theoretically unique mapping, i.e.

$$
(s)(t)(u)(s \Theta u \wedge t \Theta u \cdot \rightarrow s \overline{\bar{o}} t) .
$$

Moreover, as a set-theoretical relation, $\Theta$ can be assumed to be set-theoretically left-invariant, i.e.

$$
(s)(t)(u)(s \overline{\bar{o}} t \wedge t \Theta u \cdot \rightarrow s \Theta u) \text {. }
$$

Now, I define another relation $\Lambda$ by

$$
S \Lambda T ₹ \cdot S \Theta T \wedge \$(S) \wedge \$(T) .
$$

This relation is unique in general sence, i.e. $(S)(T)(U)(S \Lambda U \wedge T \Lambda U \cdot \rightarrow S=T)$, and also left-inveriant. These are easily provable by making use of (4.3), (7.6), and (7.8). Since $A$ and $\Theta$ are set-theoretically equivalent, i.e. $(s)(t)(s \Lambda t \equiv s \Theta t)$, so the axiom of replacement can be described as

$$
(x)(\exists y) y\left\langle\Lambda^{*} \in\right) 。 x
$$

where $A$ is left-invariant and unique.

According to (2.18), the left-invariant and unique relation $\Lambda$ can be expressed in the form $\{\Gamma)$, so the axiom of replacement can be described as

$$
(x)(\exists y) y\left\langle\{\Gamma)^{*} \in\right)_{0} x .
$$

In my system, this formula is provable without imposing any condition on $\Gamma$.

Proof of the axiom of replacement $(x)(\exists y) y\left\{\{\Gamma)^{*} \in\right)_{0} x$. For any $x$, take an object $U$ such that $U\{\{\Gamma) \in) x$ by (3.5). Next, by (3.6), take an object $Y$ such that $Y\{=(\Delta \wedge \in)) U$, where $\Delta$ is a relation defined by $T \Delta V ₹ \$(T)$.

By making use of (4.3) and (7.6), one can easily prove $(T)_{Y} \$(T)$, and $Y\{=\epsilon) Y$ holds evidently by (2.3), so $Y$ is a set according to (7.5). For any set-member $s$ of $Y$, holds $s=\in U$ by definition, so there is an object $T$ such that $s=T \in U$. Accordingly there is an object $W$ such that $T\left\{I^{\prime}\right) W \in x$ by definition. Because $\{T)$ is left-invariant by (2.8) and the member $W$ of the set $x$ is also a set by (4.4) and (7.6), $s\{\Gamma)^{*} \in x$ holds. Next, conversely, for any $s$ satisfying $s\{\Gamma)^{*} \in x$, holds $s\{\Gamma) \in x$ by (7.3), so by making use of (2.3), $s$ is a member of $Y$ by definition. Hence, $Y\left\{\{\Gamma)^{*} \in\right)_{0} x$ holds for the set $Y$.

(7.15) The fundierung axiom may be stated as that any set $m$ must be a 
null set as far as every set-member $x$ of $m$ has a common set-member with $m$. This can be described in my system shortly as

$$
(m)\left((x)_{m} x \ni^{*} \in m \rightarrow \phi(m)\right) \text {. }
$$

Proof of the fundierung axiom. For any set $m$ satisfying $(x)_{m} x \ni^{*} \in m$, $m \rho m$ is easily provable by making use of (7.3) and (7.6). Consequently, if $m$ were a non-null-object having a member $X$, then $m$ can not be regular because $m \rho m \ni X \delta m$ holds by (4.4). However, this can not hold for a set $m$, as any set must be regular by definition.

(7.16) I describe the axiom of infinity in a seemingly stronger form as follows

$$
(m)(\exists p)\left(m \in p \wedge(h)_{p}(\exists k)_{p} k\{\overline{\bar{\sigma}}){ }_{0} h\right) .
$$

Proof of the axiom of infinity. For any set $m$, take an object $R$ such that $m \in R \wedge(H)_{R}(\exists K)_{R} K\{=) H$ by (3.11). By (3.6), take next an object $P$ such that $P\{=(\Gamma \wedge \in)) R$, where $\Gamma$ is a relation defined by $S \Gamma T ₹ \$(S)$.

$(X)_{p} \$(X)$ is provable by making use of (4.3) and (7.6), and $P(=\in) P$ is provable by (2.3). Hence, $P$ is a set by (7.5). Evidently, $m \Gamma R$ holds, so $m$ is a member of $P$.

I will prove next $(h)_{p}(\exists k)_{p} k\{\bar{z})_{0} h$. Namely, for any set member $h$ of $P$, there is an object $G$ satisfying $h=G \in R$ by definition, and this $G$ must be a set according to (2.3), (4.3), and (7.6). For the member $G$ of $R$, there is a member $K$ of $R$ satisfying $K\{=) G$. For this $K,(X)_{K} \$(X)$ is provable by making use of (4.3) and (7.6), and $K\{=\in) K$ is provable by (2.3). Hence, $K$ is also a set, which implies $K \Gamma R$ by definition of $\Gamma$. Since $K=K(\Gamma \wedge \in) R$ holds by (2.3), $K$ is a member of $P$.

According to (2.3), (2.5), and (2.9), $K\{=) G$ implies $K\{=) h . \quad K\{\overline{\bar{o}})_{\circ} h$ can be derived from $K\{=) h$ by employing (7.3) and (7.8).

\section{REFERENCES}

[1] Bernays, P. [1], A system of axiomatic set theory I-VII, J. Symb. Log.; I, vol. 2 (1937), 65-77; II, vol. 6 (1941), 1-17; III, vol. 7 (1942), 65-89; IV. vol. 7 (1942), 133-145; V, vol. 8 (1943), 89-106; VI, vol. 13 (1948), 65-79; VII, vol. 19 (1954), 81-96.

[2] Fraenkel, A., [1] Untersuchungen über die Grundlagen der Mengenlehre, Math. Ztschr., vol. 22 (1925), 250-273.

[3] Fraenkel, A., [2] Einleitung in die Mengenlehre, 3rd. ed., Berlin (1928).

[4] Gandy, R. O., [1] On the axiom of extensionality I, II, J. Symb. Log.; I, vol. 21 (1956), 
36-48; II, vol. 24 (1959), 287-300.

[5] Gödel, K., [1] The consistency of the axiom of choice and of the generalized continuum hypothesis with the axioms of set-theory, Annals of Math. Studies, No. 3, Princeton (1940).

[6] v. Neumann, J., [1] Die Axiomatisierung der Mengenlehre, Math. Ztschr., vol. 27 (1928), 669-752.

[7] v. Neumann, J., [2] Über eine Widerspruchsfreiheitsfrage in der axiomatischen Mengenlehre, J. f. reine u. angw. Math., vol. 160 (1929), 227-241.

[8] Ono, K., [1] A set theory founded on unique generating principle, Nagoya Math. J., vol. 12 (1957), 151-159.

[9] Ono, K., [2] A theory of mathematical objects as a prototype of set theory, Nagoya Math. J., vol. 20 (1962), 105-168.

[10] Ono, K., [3] On a practical way of describing formal deductions, Nagoya Math. J., vol. 21 (1962), 115-121.

[11] Ono, K., [4] A stronger system of object theory as a prototype of set theory, Nagoya Math. J., vol. 22 (1963), 119-167.

[12] Quine, W. V. O., [1] New foundations for mathematical logic, Am. Math. Monthly, vol. 44 (1937), 70-80.

[13] Quine, W. V. O., [2] Mathematical logic, Cambridge Mass. (1940, revised ed. 1951).

Mathematical Institute

Nagoya University 